the phosphorylation of the purified bovine brain catalytic subunit (Fig. 3 of ref. 1) leads to its activation.

In intact cells where both the cyclic AMP pathway and the PKC pathway have been shown to function, it has been shown that the link between the two pathways is most probably $G_{i}$ (the inhibitory GTP protein of cyclase) $)^{2}$. Potentiation of adenylate cyclase by the PKC pathway results from the phosphorylation of $G_{i}$, thus relieving the inhibitory tonus on adenylate cyclase $e^{2.3}$. The effect of phorbol esters is quantitatively similar to that of pertussis toxin ${ }^{2}$. Yoshimasa et al. ${ }^{1}$ did not examine the $G_{i}$ component in their system .

Another major consideration is the possibility that the phosphatidylinositolbisphosphate breakdown pathway and the adenylate cyclase pathway interact through the mobilization of $\mathrm{Ca}^{2+}$ and not only through the activation of PKC. The ample evidence on the activation of adenylate cyclase by $\mathrm{Ca}^{2+}$-calmodulin, both in the central nervous system and peripheral tissues (for example, ref. 4) should alert us to the possibility that inositol trisphosphate-induced mobilization of $\mathrm{Ca}^{2+}$ could lead to activation of calmodulin-sensitive adenylate cyclase. Attractively, the mode of activation is transient, the property of a true signal. Simultaneous activation of PKC on phosphatidylinositol bisphosphate breakdown can subsequently result in the inhibition of calmodulin-dependent adenylate cyclase ${ }^{5}$, providing long-term inhibition.

Alexander Levitzki Department of Biological Chemistry, Institute of Life Sciences,

The Hebrew University of Jerusalem, Jerusalem 91904, Israel

. Yoshimasa, T.. Sibley. D.R., Bouvier, M. Lefkowitz, R.J. \& Caron, M.G. Nature 327, 67-70 (1987).

2. Johnson, J.A., Goka, T.J.\& Clark, R.B. J. cyclic Nucleotide Protein Phosphor. 11, 199-215 (1986).

3. Bell, J.D. \& Brunton, L.L.J. biol. Chem. 261, 12036-12041 (1986).

4. Livingstone, M.S., Sziber, P.P. \& Quinn, W.G. Cell 37, $205-215$ (1984).

5. Brostom, M.A. et al. J. biol.Chem. 257, 6758-6765 (1982)

\section{Second messengers or second agonists?}

SIR-In the recent article by Piomelli et $a l^{\prime}$, an unidentified lipoxygenase metabolite was described as a "second messenger" in presynaptic inhibition of Aplysia sensory neurons by FMRFamide. By definition, a second messenger is the first intracellular signal generated in response to an intercellular primary messenger, such as a hormone or neurotransmitter (model II). This definition raises two important issues: the number of steps between activation of FMRFamide receptors and production of active lipoxygenase mediators; and the distinction between intracellular and extracellular targets of the mediators. An alternative hypothesis, (model I) consistent with the authors' own data and for which there are ample biological precedents, is that a lipoxygenase metabolite, possibly the downstream product of another messenger pathway, may act at the cell surface through its own receptor as a second agonist (see figure).

On the first issue, the first documented products of stimulation are 5- and 12-HETEs (5- and 12-hydroxyeicosatetraenioc acids) derived from arachidonic acid, suggesting that a spectrum of candidate mediators are generated by a FMRFamide-regulated biochemical cascade. Identification of the signal among the various products was undertaken by comparing the actions of synthetic eicosanoids to that of FMRFamide. Both arachidonic acid and lipoxygenase pathway-derived products mimic the action of FMRFamide in some, but it must be emphasized, not all respects. However, because the results come exclusively from experiments using extracellular application of these agents, the argument for an essential intracellular action of a lipoxygenase product must be carried by the indirect, pharmacological evidence. One reagent, $p$-bromophenacyl bromide, is described as a "phospholipase A2 or diacylglycerol lipase" inhibitor, but is in fact a non-specific histidine or carboxylic acid-modifying agent, and its specificity and ability to cross the plasma membrane in whole living cells is unknown. A selective effect of this reagent on lipid lipases would have been more convincing if

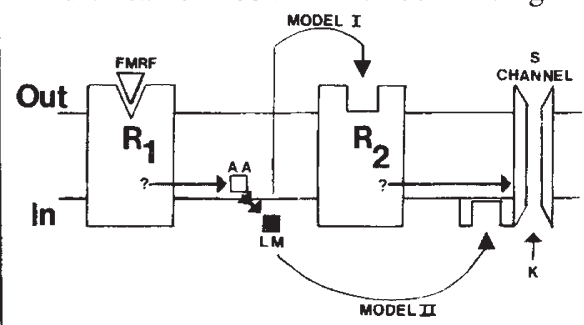

responses to the products of lipase action (for example arachidonic acid,12-HPETE (12-hydroperoxyeicosatetraenoic acid) were unchanged after treatment. NDGA (nordihydroguaiaretic acid) is also both a cyclo- and lipoxygenase inhibitor in mammalian neutrophils ${ }^{2}$, and may have similar complexities in Aplysia neurons. The data therefore are necessary but not sufficient to establish that the action of FMRFamide on the $\mathrm{S}$ channel is directly due to intracellular activity of a lipoxygenase mediator.

The functions and modes of action of eicosanoids have been examined in most detail in vascular and immune cells, which have cell-surface receptors for both cycloand lipoxygenase-derived metabolites ${ }^{3.4}$. There is little evidence for intracellular targets for these classes of compounds.

In the platelet, activation of several distinct receptors initiates a cascade of events, including the production of eicosanoids such as thromboxane A2. For example, platelet-activating factor (PAF) stimulates the hydrolysis of inositol phospholipids and evidence indicates that arachidonic acid is also released. Furthermore, thromboxane A2 amplifies the response to PAF by acting on its own surface receptor to stimulate further inositol lipid hydrolysis. If platelets can be used as a model for Aplysia neurons, then the second messenger that ultimately causes arachidonic acid release and its subsequent metabolism to active products remains unknown, and a lipoxygenase metabolite may act extracellularly through its own receptor to control channel activity.

We feel caution is required in causeand-effect interpretations of the application of non-selective lipase-blocking agents and broad spectrum cyclo- and lipoxygenase inhibitors to intact cells.

Michael R. Hanley

MRC Molecular Neurobiology Unit,

University of Cambridge,

Hills Road, Cambridge CB2 2QH, UK

C. Peter Downes

S. K. \& F. Research Ltd.,

The Frythe, Welwyn,

Hertfordshire AL69AR, UK

1. Piomelli, D. et al. Nature 328, 38 (1987)

Billan, M et al J biol. Chem. 260, 6899 (1985)

3. Armstrong, R. et al. Br. J. Pharmac. 79, 953 (1983).

4. Mong. S. et al. Molec. Pharmac. 30, 235 (1986).

\section{Anucleolate mutant due for re-examination}

SIR-I was intrigued to read, in the account of recent research on the structure and function of the nucleolus by Gwyn Jordan', the current idea that the fibrillar centres represent the pre-initiation complexes of ribosomal RNA genes. My interest stems from the fact that, in 1965 , I described ${ }^{2}$ similar structures in the nuclei of the anucleolate mutant of Xenopus and suggested, on ultrastructural grounds, that they might be homologous with what are now referred to as fibrillar centres. If this interpretation is correct, these regions obviously can exist in the absence of ribosomal DNA. I would be curious to know whether anyone has recently examined this mutant with the newer techniques summarized by Jordan. If not, such a re-examination might help to resolve this interesting question.

Genetics Department,

University of Edinburgh,

Edinburgh EH9 $3 J N$, UK

1. Jordan, G. Nature 329, 489 (1987).

2. Jones, K.W. J. ultrastruct. Res. 13, 257 (1965)

\section{Scientific Correspondence}

Scientific Correspondence is intended to provide a forum in which readers may raise points of a scientific character. They need not arise out of anything published in Nature. In any case, priority will be given to letters of fewer than 500 words and five references. 